\title{
La Vie de la SALF
}

\section{SOCIÉTÉ D’ANDROLOGIE DE LANGUE FRANÇAISE}

\section{NOUVEAUX STATUTS ADOPTES LE 10 DECEMBRE 1998}

\section{OBJET - DÉNOMINATION SOCIALE}

\section{Article 1:}

Il est fondé entre les adhérents aux présents statuts une association régie par la loi du 1er juillet 1901 et le décret du 16 août 1901, ayant pour titre «Société d'Andrologie de Langue Française».

\section{Article 2:}

L'association dite «Société d'Andrologie de Langue Française» a pour but d'encourager, de promouvoir et de réaliser toute action pouvant favoriser :

- l'étude de la fertilité, de la stérilité et de la contraception masculine,

- la recherche clinique et fondamentale dans les domaines de la physiologie, de la biologie et de la pathologie de l'appareil génital mâle,

- la connaissance des particularités physiologiques, pathologiques et psychologiques du sexe masculin,

- la diffusion des informations dans le domaine de l'Andrologie (réunions, conférences, publications).

- et de contribuer à l'enseignement de l'Andrologie et à la formation continue des praticiens de santé.

\section{Article 3 : Siège Social :}

Le siège de l'association est :

8, esplanade Compans Caffarelli - 31080 TOULOUSE CEDEX

Le Conseil d'Administration est habilité à décider du changement de siège social à la seule réserve que le dit siège soit situé sur un territoire métropolitain.

Article 4: Durée - Dissolution

La durée de l'association est fixée à 50 (cinquante) années à compter de la déclaration qui sera faite conformément à la loi du 1er juillet 1901.

Cette durée sera prorogée, éventuellement, en temps voulu par décision de l'assemblée générale ordinaire ou extraordinaire.

L'assemblée extraordinaire est également habilitée à prononcer la dissolution de l'association.

Article 5: Membres de l'association

Seules les personnes physiques peuvent être membre de l'Association.

L'association se compose de membres et de membres honoraires. Les membres et membres honoraires jouissent de la totalité des droits découlant de leur appartenance à l'association dans la mesure où ils respectent LA CHARTE réalisée par l'association. 
L'admission à titre de membre de l'association est subordonnée à l'envoi d'une demande écrite au Président de l'Association. Les demandes d'admission devront mentionner obligatoirement le nom de deux parrains déjà membres de l'association. Le Conseil d'Administration à la majorité simple statue sur l'admission sans avoir à justifier sa décision. Il en est de même en cas de refus de la demande d'admission.

Les membres honoraires sont des personnes physiques choisies à la majorité simple par le Conseil d'Administration pour leur compétence reconnue dans le domaine scientifique. Les membres honoraires sont admis pour une durée limitée.

Les membres doivent payer une cotisation annuelle dont le montant est fixé chaque année par l'assemblée générale de l'association. Les membres honoraires sont dispensés de tout versement de cotisation.

\section{Article 6: Cessation d'appartenance}

Cessent de faire partie de l'association, sans que leur départ puisse mettre fin à celle-ci :

$1^{\circ}$ - ceux qui auront donné leur démission par lettre adressée au Président et dont la démission aura été acceptée.

$2^{\circ}$ - ceux qui auront été radiés par le Conseil d'Administration pour infraction aux présents statuts ou pour motif grave, quinze jours après avoir été mis en demeure, par lettre recommandée, de fournir leurs explications soit écrites, soit orales. La décision sera notifiée au membre exclu par lettre recommandée, dans la huitaine qui suit la décision. Cette décision est sans appel.

$3^{\circ}$ - les membres décédés, sans préjudice du paiement par leurs héritiers des sommes dues pour cotisations au jour du décès, y compris la cotisation de l'année courante.

$4^{\circ}$ - les membres qui, après mise en demeure restée infructueuse, n'auront pas payé pendant deux années consécutives leur cotisation.

Article 7 : Responsabilité des membres

Les membres sont soumis à la responsabilité de droit commun en matière d'association. Pour les administrateurs, il ne pourra y avoir aucune limitation de responsabilité de leur fait personnel.

\section{II - RESSOURCES DE L'ASSOCIATION}

\section{Article 8:}

Les ressources de l'association sont définies par :

- des cotisations versées par les membres,

- des intérêts et des revenus des biens et valeurs appartenant à l'association,

- des autres ressources autorisées par la loi.

- des organisations de congrès, de l'enseignement et de la formation.

\section{Article 9:}

Dans le cadre de la gestion de l'association, l'Assemblée Générale désigne en son sein un Trésorier élu pour une durée indéterminée à la majorité requise en matière d'assemblée générale ordinaire. Le Trésorier est révocable par les membres en assemblée générale ordinaire dans les mêmes conditions que son élection.

Le Trésorier a au même titre que le Président la signature bancaire, qui pourra dans des conditions limitées et temporaires en cas d'empêchement être déléguée au Secrétaire du Conseil d'Administration.

Le Trésorier assure la gestion quotidienne de l'association sous la responsabilité du Président. Il tient au jour le jour une comptabilité deniers par recettes et par dépenses ; à la fin de l'exercice comptable, il est tenu compte de tous les produits et charges qui s'y rattachent. Il pourra être assisté dans cette mission par un expert comptable.

Il participe aux réunions du Conseil d'administration sans avoir voix délibérative.

Il présente chaque année au Conseil d'Administration et à l'Assemblée Générale Annuelle les comptes de l'exercice social de l'association qui commence le 1er décembre et se termine le 30 novembre. Ces comptes 
arrêtés préalablement par le Conseil d'Administration sont soumis à l'adoption de l'assemblée générale annuelle.

Article 10:

Les cotisations sont dues dès l'admission définitive et correspondent à l'année sociale en cours, sans fractionnement d'aucune sorte.

Leur montant est fixé et modifié sur proposition du Conseil d'Administration comme indiqué à l'article 21.

Les membres honoraires sont dispensés de tout versement, de toute cotisation et de toute prestation en nature. Ils consentent leur appui à l'association.

\section{III - ADMINISTRATION}

Article 11 : Conseil d'Administration

Le Conseil d'Administration comprend :

- 15 membres élus au scrutin secret par l'assemblée générale ordinaire à la majorité simple des membres présents.

- les Administrateurs sont élus pour une durée de 3 ans non renouvelable. Les anciens administrateurs devront attendre 1 an après la fin de leur mandat pour présenter leur candidature à l'assemblée générale.

Un tiers des Administrateurs est renouvelable chaque année.

Les membres demeurent libres de se présenter au cours de l'Assemblée Générale et au plus tard avant le vote sur l'élection des administrateurs. Les membres qui présentent leur candidature au poste d'administrateur devront être obligatoirement présents à l'assemblée générale.

En cas de démission d'un administrateur, il est procédé à son remplacement définitif par la plus prochaine assemblée générale.

Si le nombre d'administrateurs devient inférieur à 11 , le conseil ne peut plus prendre de décisions à peine de nullité. Le Président doit convoquer dans le mois qui suit une assemblée générale réunie extraordinairement pour procéder à l'élection des nouveaux membres.

Article 12: Pouvoirs du Conseil

Le Conseil d'Administration est investi des pouvoirs les plus étendus pour prendre toutes décisions qui ne sont pas réservées à l'assemblée générale. Il se prononce souverainement sur toutes les admissions et radiations des membres de l'association. Il propose à l'assemblée le règlement intérieur.

Article 13: Bureau

Le Conseil d'Administration constitue un bureau comprenant au minimum un Président et un Secrétaire Général choisis parmi les administrateurs élus par vote à la majorité des membres, les candidats ne prennent pas part au vote les concernant.

Le mandat du Président devra être d'une durée minimale de 2 ans et maximale de 3 ans. En aucune manière la durée de sa fonction ne pourra être prorogée.

Article 14: Rôle du Président

Le Président représente l'association dans tous les actes de la vie civile et est investi de tous pouvoirs à cet effet.

Il a notamment qualité pour ester en justice comme défendeur au nom de l'association et comme demandeur avec l'autorisation du Conseil d'Administration. Il ne peut transiger en tout cas qu'avec l'autorisation du Conseil d'Administration.

Il préside toutes les assemblées générales. En cas d'absence, il sera remplacé par le Secrétaire Général. 
Le Secrétaire Général convoque les Assemblées Générales et les réunions du Conseil d’Administration en accord avec le Président.

Il est chargé de tout ce qui concerne la correspondance et les archives.

Il rédige les procès verbaux des réunions, délibérations ou assemblées et, en général, toutes les écritures concernant le fonctionnement de l'association à l'exception de celles qui concernent la comptabilité.

Il tient le registre spécial prévu à l'article 5 de la loi du 1er juillet 1901 et les articles 6 et 31 du décret du 16 août 1901. Il assure l'exécution des formalités prescrites par les dits articles.

\section{III - ASSEMBLÉES}

\section{Article 16: Assemblée Générale}

L'Assemblée Générale se compose de tous les membres à jour de leur cotisation et des membres honoraires de l'association.

\section{Article 17:}

Les assemblées sont ordinaires ou extraordinaires.

L'assemblée générale ordinaire a lieu 1 (une) fois par an. La date exacte en est arrêtée par le Conseil d'Administration au moins 30 (trente) jours à l'avance.

L'assemblée générale ordinaire peut être convoquée extraordinairement en cas de circonstances exceptionnelles par le Président après accord du Conseil d'Administration ou sur demande écrite, déposée au secrétariat, d'un cinquième au moins des membres.

Dans ce dernier cas, la réunion doit avoir lieu dans les 30 (trente) jours qui suivent le dépôt de la demande au secrétariat.

Pour toutes les assemblées, les convocations doivent être envoyées au moins 15 (quinze) jours à l'avance et indiquer l'ordre du jour. Avec la convocation est joint un pouvoir, tout membre pouvant se faire représenter par un autre membre. Chaque membre présent ne pourra être titulaire de plus de deux pouvoirs. En envoyant un pouvoir en blanc, tout membre émet un vote favorable à l'adoption des points prévus à l'ordre du jour de la convocation de l'Assemblée, et un vote défavorable à l'adoption de toutes les autres décisions. Les pouvoirs en blanc devront être reçus au plus tard la veille de la réunion de l'Assemblée Générale.

En début de séance de l'Assemblée Générale, une feuille de présence est dûment émargée par les membres présents ou représentés. Les pouvoirs donnés aux membres sont annexés à cette feuille de présence.

L’Assemblée est présidée dans les conditions fixées à l'article 14. Le Président de séance est assisté d'un bureau composé de deux scrutateurs et d'un secrétaire désigné par l'assemblée préalablement à l'examen de l'ordre du jour.

\section{Article 18: Ordre du jour}

Les sujets portés à l'ordre du jour sont déterminés par le Conseil d'Administration qui s'autorise à entendre toute personne appartenant ou non à l'association, sur tout sujet qui lui semble d'intérêt.

Outre les sujets portés à l'ordre du jour par le Conseil d'Administration, toute proposition portant la signature de trois membres et déposée au secrétariat au moins huit jours avant la réunion, sera soumise à l'assemblée. 


\section{Article 19: Assemblée Générale Ordinaire}

L'assemblée générale ordinaire est réunie au moins une fois l'an. Elle reçoit le compte-rendu des travaux du Conseil d'Administration et les comptes du Trésorier.

Elle statue sur leur approbation.

Elle statue sur le règlement intérieur de l'association.

Elle statue souverainement sur toutes les questions relatives au fonctionnement de l'association, donne toute autorisation au Conseil d'Administration, au Président, au Secrétaire Général, au Trésorier pour effectuer toutes opérations entrant dans l'objet de l'association et qui ne sont pas contraires aux dispositions de la loi du 1er juillet 1901 pour lesquelles les pouvoirs qui leur sont conférés par les statuts ne seraient pas suffisants.

Elle vote le budget de l'année.

Elle ratifie la fixation du montant de la cotisation annuelle.

Toutes les délibérations et décisions de l'assemblée générale sont prises à la majorité des membres présents.

\section{Article 20: Assemblée Générale Extraordinaire}

L'assemblée générale extraordinaire statue dans les conditions suivantes :

Elle peut apporter toutes modifications aux statuts.

Elle peut ordonner la prorogation ou la dissolution de l'association ou sa fusion avec toute autre association poursuivant un but analogue ou son affiliation à toute union d'associations mais dans ces divers cas, elle doit être composée de la moitié des membres ayant le droit de prendre part aux assemblées.

$\mathrm{Au}$ cas où le quorum ne serait pas atteint, il sera convoqué dans les 15 (quinze) jours une nouvelle assemblée générale. Les décisions seront alors prises à la majorité des membres présents ou représentés.

\section{Article 21:}

Les délibérations des assemblées sont consignées par le Secrétaire Général sur un registre et signées par les deux scrutateurs et le secrétaire. Ces procès verbaux constatent le nombre de membres présents aux assemblées générales.

Les délibérations du Conseil d'Administration sont consignées sur un registre par le Secrétaire Général et signées par lui et par le Président. Il est également tenu un registre de présence au Conseil.

\section{Article 22:}

En cas de dissolution volontaire ou forcée, l'assemblée générale extraordinaire statue sur la dévolution du patrimoine de l'association, sans pouvoir attribuer aux membres de l'association autre chose que leurs rapports. Elle désigne les établissements publics ou les établissements privés poursuivant un but similaire qui recevront le reliquat de l'actif après paiement de toutes dettes et charges de l'association et de tous frais de liquidation. Elle nomme pour assurer les opérations de liquidation, un ou plusieurs commissaires aux comptes qui seront investis à cet effet de tous pouvoirs nécessaires.

\section{Article 23:}

Le Président, au nom du Conseil d'Administration, est chargé de remplir toutes les formalités de déclaration et de publication prévues par la loi du ler juillet 1901 et par le décret du 16 août 1901. 
Article 26: Contestation

Toutes les contestations qui pourraient s'élever pendant la durée de l'Association ou lors de sa liquidation, entre l'Association et ses membres ou ses administrateurs, seront jugées conformément à la loi et soumises à la juridiction des tribunaux compétents.

\section{Article 27: Règlement intérieur}

La société est pourvue d'un règlement intérieur fixant les modalités pratiques d'application des statuts de l'association.

Ce règlement intérieur est étudié et révisé s'il y a lieu par le Conseil d'Administration. Son contenu est proposé au vote de l'assemblée générale et adopté par elle. Toute modification doit être ratifiée par l'assemblée générale avant sa mise en application. En cas de nécessité impérative, le conseil peut prendre toute décision nécessitée par les circonstances. Ces décisions ne sont applicables qu'à titre précaire et doivent être ratifiées lors de la prochaine assemblée générale.

\section{La Société d'Andrologie de Langue Française ouvre son site Internet L'adresse en est :}

\section{http://salf.univ-lyon1.fr/salf}

\section{Ce site comporte les rubriques suivantes :}

- Informations générales sur la SALF

- Calendrier des Manifestations de la Société

- Sommaires de la revue Andrologie

- Publications marquantes en Andrologie

- Annuaire des membres de la Société *

- Enseignements de l'Andrologie

- Questions / Réponses en Andrologie

- Un lien avec le site internet de l'International Society of Andrology

- Envoyer un message au site de la SALF

- Comment adhérer à la SALF ?

* L'annuaire des membres de la de la SALF est en cours de confection :

il pourra indiquer pour chaque membre de la société : titre, nom, prénom, adresse professionnelle, téléphone, télécopie, adresse électronique, mots-clés résumant l'activité professionnelle.

Sa diffusion sur le site internet a fait l'objet d'une déclaration à la CNIL, la mise à jour de l'annuaire implique que chaque membre de la SALF donne autorisation à la société de divulguer les données le concernant, à l'aide du formulaire suivant: 


\section{Mise à jour de l'annuaire de la Société d'Andrologie de Langue Française}

La Société d'Andrologie de Langue Française diffuse sur son site internet un annuaire des membres de la société.

Il comporte le titre universitaire, le nom, le prénom, l'adresse professionnelle, les $\mathrm{n}^{\circ}$ de téléphone, de télécopie, l'adresse électronique et des mots-clés résumant l'activité professionnelle des membres de la société.

Si vous en êtes d'accord, nous vous demandons de nous communiquer les renseignements vous concernant par courrier électronique à l'adresse du site internet de la SALF : salfweb@rockefeller.univ-lyon1.fr ou de remplir l'autorisation ci-dessous et l'adresser au Dr. Hervé LEJEUNE, Clinique Endocrinologique, Hôpital de l'Antiquaille, 1 rue de l'Antiquaille, 69321 LYON cedex 05:

J'autorise la Société d'Andrologie de Langue Française à faire apparaître dans l'annuaire des membres de la société, figurant sur le site internet :

\section{http://salf.univ-lyon1.fr/salf}

les informations suivantes me concernant:

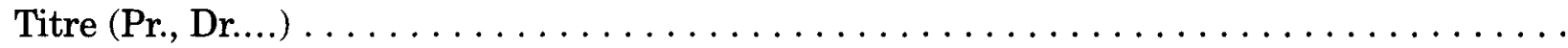

Nom et Prénom :

Adresse professionnelle :

Téléphone :

Télécopie :

Adresse électronique (un lien pourra être établi entre le site et votre adresse e-mail) :

Spécialité d'exercice médical et/ou Mots-clés résumant l'activité professionnelle :

date :

signature :

Vous disposez d'un droit d'accès, de modification, de rectification et de suppression des données qui vous concernent (art. 34 de la loi "Informatique et Libertés" du 6 janvier 1978). Pour exercer ce droit, contacter le site à son adresse e-mail salfweb@rockefeller.univ-lyon1.fr ou contacter le Dr. Hervé Lejeune, Clinique Endocrinologique, Hôpital de l'Antiquaille, 1 rue de l'Antiquaille, 69321 LYON cedex 05, Tel. 0472385186 , Fax 0472385071 